\title{
Systems Concurrent Engineering for the Conception of a Hybrid Vehicle
}

\author{
Geilson Loureiro $^{\mathrm{a}, 1}$, Jonas Bianchini Fulindi ${ }^{\mathrm{b}}$, Letícia Azevedo de Oliveira Fideles ${ }^{\mathrm{c}}$, \\ Daniella Fernandes ${ }^{d}$, Rosely Semabukuro ${ }^{e}$, and Carlos de Oliveira Lino ${ }^{\mathrm{f}}$ \\ ${ }^{a}$ Technologist and Professor at the Integration and Testing Laboratory, Brazilian \\ Institute for Space Research, INPE (São José dos Campos), Brazil. \\ ${ }^{\mathrm{b}}$ Post graduate student at Brazilian Institute for Space Research - INPE. \\ c,d,e,f Post graduate students at Technological Insitute of Aeronautics - ITA.
}

\begin{abstract}
This paper presents a systems concurrent engineering approach for the conception of a hybrid vehicle. Traditional approaches focuses on the product, development organization and the product concepts of operation (CONOPS). In those approaches the overall view of the inherent complexity in the development of a product, its life cycle processes and their performing organizations are not taken into consideration. The systems concurrent engineering performs stakeholder analysis, requirements analysis, functional analysis and implementation architecture analysis, simultaneously, for the product, its life cycle processes and their performing organization. From the analysis, requirements and attributes are captured for the product and its life cycle processes organizations and the relationship among them are identified. Conclusions are that impact, traceability and hierarchy links promote the anticipation of life cycle process requirements to the early stages of systems architecting. Late changes are avoided, development costs are dramatically reduced while satisfaction of stakeholders over product life cycle is increased.
\end{abstract}

Keywords. Systems concurrent engineering, systems engineering, concurrent engineering, complex product, integrated product development.

\section{Introduction}

Hybrid systems incorporate two sources of energy in a single vehicle, combining an internal combustion engine and an electric motor. In the hybrid vehicles currently on the market, the internal combustion engine can directly trigger the wheels or an electric generator to charge a battery that feeds an electric motor.

This paper aims to present a systems concurrent engineering approach for the conception of a hybrid vehicle. The approach is different from traditional systems engineering approach because it anticipates to the early stages of system

\footnotetext{
${ }^{1}$ Technologist and Professor at the Integration and Testing Laboratory, Brazilian Institute for Space Research, Av. dos Astronautas 1758, São José dos Campos, Brasil; 12227-010; Tel: +55 (12) 39456317; Fax: +55 (12) 39411884; Email: geilson@lit.inpe.br
} 
architecting the product life cycle process requirements. It proposes to simultaneously develop, from the outset, the product and its life cycle processes performing organizations.

The paper is organized as following: Section 2 presents the traditional systems engineering and concurrent engineering approaches. Section 3 presents the systems concurrent engineering approach framework and method. Section 4 presents the models derived for the hybrid vehicle using the approach. Section 5 discusses the advantages and opportunities for improving the proposed approach. Section 6 concludes this paper.

\section{Traditional systems engineering and concurrent engineering}

Automotive products are complex. They are multidisciplinary products, they must cope with extreme environmental conditions over their life cycle (vibration, temperature range, altitude range, electromagnetic interference and compatibility), they must undergo very strict calibration and tuning procedures. Automotive development organizations are worth the order of billion dollars. A car may take from a year to four years to develop. There are many opportunities to improve productivity over a car life cycle if a concurrent engineering approach takes place from the beginning of the car architecting stage.

Traditional systems engineering approaches do not provide an overall view of the system during its various life cycle processes. They focus on an operational product development starting from product concept of operations. They also focus on the development organization that must be put in place in order to assure that the product meets its operational requirements $[2,3,6,8]$. A product has life cycle processes other than operations and it must be recognized from the outset in order to promote gains in productivity in the product development organization, by the avoidance of late changes, and in other product life cycle process organizations, as the product will be developed taking into consideration their requirements. Life cycle process organizations themselves can be developed simultaneously to product development, when they are part of the scope of the whole product development effort.

For example the NASA systems engineering handbook [8] states that systems engineering focuses in the development and the realization of a final product. Modern commercial standards, such as EIA 632 [2], state that systems engineering focuses on the operations product and on capturing requirements for the other product life cycle processes. In other words, these requirements are captured not to impact product development. The product will be systems engineered with operations in mind. When its architecture (and maybe detailed design) is defined, then life cycle processes requirements are captured to be implemented in life cycle process performing organizations. This paper proposes a method to take into consideration the impact of these organizations on the product during the product architecting process.

Conceptually, concurrent engineering acknowledges benefits of anticipating life cycle process requirements to the early stages of product development. For space products, these early stages are the system architecting phases. A systems 
approach requires life cycle process requirements to be balanced in the beginning of the product development process. Concurrent engineering, however, in practice, treats life cycle processes separately and optimizes product design seeking each life cycle process productivity increase. For example, DFA optimizes for assemblability, QFD, for customer satisfaction, DFI, for inspectability, and so on. Also, concurrent engineering is, in practice, applied to parts design and not to systems composed of many integrated parts [5]. This paper proposes how the concurrent engineering concept can be used for systems engineering.

\section{The systems concurrent engineering approach}

Hitchins [4] states that complexity can be understood by what he calls complexity factors. They are variety, connectedness and disorder. Variety accounts for the number of different elements you have in a set. Regarding products, variety refers, for example, to the number of different parts a product may have, number of different functions it accomplishes, number of different requirements categories it is supposed to meet, number of different stakeholders it should satisfy. Connectedness refers to the relationships among elements. For example, how parts interact, how functions affect one another, how requirements conflict to each other, how value flow among stakeholders. Disorder refers to the level of tangling of those relationships. For example, is there a structure pattern of deploying stakeholder requirements through functional concept up to implementation architecture?

Figure 1 presents a framework to address complexity in product development the total view framework evolved from Loureiro [7]. It has three dimensions. Each dimension addresses one of the complexity factors mentioned above. The analysis dimension addresses the variety factor. Along the analysis dimension, it is deployed what must be analysed in order to develop a complex product. A systems engineering process consists of stakeholder analysis, requirements analysis, functional analysis and implementation or physical analysis. The integration dimension addresses the connectedness factor. It defines what must be integrated along an integrated product development process: product elements and organization elements. Organization here refers to the organizations that perform product life cycle processes. Product elements and organization elements are the system elements. The structure dimension addresses the disorder factor. According to Alexander [1] all structures evolve into a hierarchy. System breakdown structures are also represented in hierarchies.

Figure 2 provides an overview of a method within the total view framework. The method is called concurrent structured analysis method evolved from Loureiro [7]. Stakeholder analysis, requirements analysis, functional analysis and implementation (or physical) analysis is performed, simultaneously, for the product under development and its life cycle process performing organizations. The analysis processes are performed at each layer of the system breakdown structure. For example, if a car is the product under development, the analysis processes are performed at the car layer, at the powertrain layer, at the engine layer and so on. 
G. Loureiro et al.

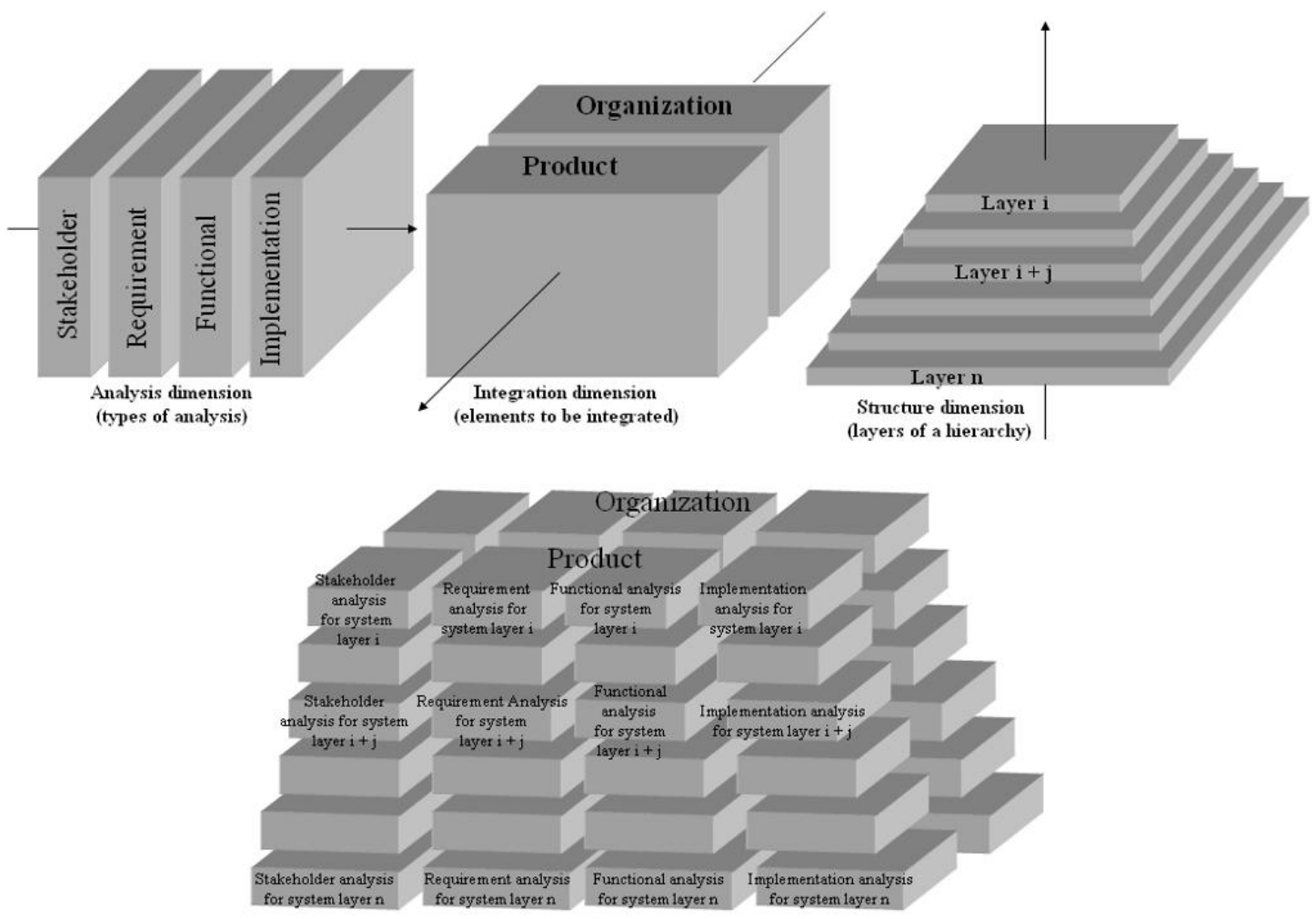

Figure 1. A framework to address complexity in complex product development - the total view framework

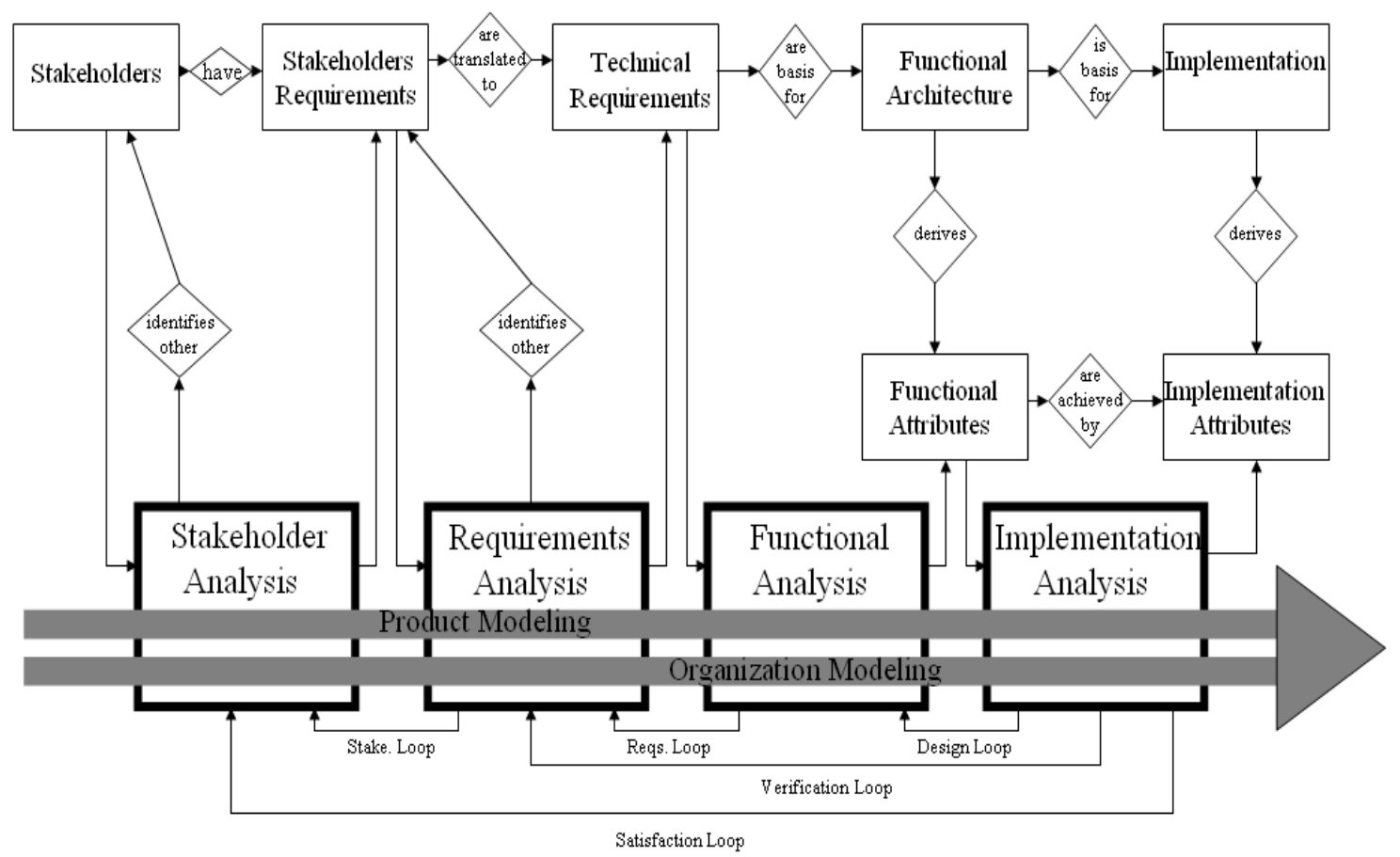

Figure 2. A method within the total view framework - the concurrent structured analysis method 
Figure 3 details the concurrent structured analysis method showing how to incorporate the concurrent engineering concept in the systems engineering process:

Step 1: Identify the product mission, the product life cycle processes and their scenarios and, the scope of the development effort. Product mission refers to the product purpose or reason of being. Life cycle process scenarios are the alternatives in each process (for example, preventive or corrective maintenance) or the decomposition of a process (for example, advanced technology development, process engineering as components of the development process). The scope of the development effort consists of the life cycle processes or their scenarios that the development organization is also responsible for accomplishing. For example, EMBRAER is responsible for developing aircraft but is also responsible for providing maintenance services.

Step 2: Identify product stakeholders and their concerns for each product life cycle process scenario. Product stakeholders are the people who affect or are affected by the product during its life cycle. Product stakeholders are identified per life cycle process scenario. Identify organization stakeholders and their concerns for each process within the scope of the development effort. Organization stakeholders are the people who affect or are affected by the business of the organization in question. Organization stakeholders are identified per life cycle process scenario within the scope of the development effort. From stakeholder concerns, stakeholder requirements are identified and measures of effectiveness (MoEs) are derived. MoEs must measure how the system meets the stakeholder requirements. From stakeholder requirements, functions, performance and conditions are identified. The definition of what functions the system will perform, how well the system is going to perform such functions and under which conditions comprise the requirements analysis process. Requirement analysis transforms stakeholder requirements into system requirements. System requirements will be met not only by product elements but also by organization elements.

Step 3: Identify functional context for product at each life cycle process scenario and for organization at each life cycle process scenario within the scope of the development effort. Functional context defines the function performed by the system element and identifies the elements in the environment of the system. The environment of the system contains the elements outside the system function scope and that exchanges material, information and energy flows with the system. Those flows define logical interface requirements. Environment elements may have different relevant states. Sets of environment element states are called circumstances. The system must have different modes depending on the circumstances. Behaviour modelling is required to show under which conditions system mode and system state transition occurs. Functions are identified per mode. Functions are identified from outside in by identifying which responses the system is supposed to give to deal with each stimulus provided by the environment elements. For each function, performance requirements are identified. Circumstances, flows between the system and the environment and function failures are sources of hazards. Risk analyses are performed on each identified potential hazard and exception handling functions are also identified at this stage. 
Step 4: Identify implementation architecture context for product at each life cycle process scenario and for organization at each life cycle process scenario within the scope of the development effort. Physical connections between the system and the environment elements define the physical external interface requirements. Physical parts are identified. Physical internal interfaces are defined by architecture connections and architecture flows among those parts. Allocation matrix relates physical parts and physical interfaces to the functions and functional flows.

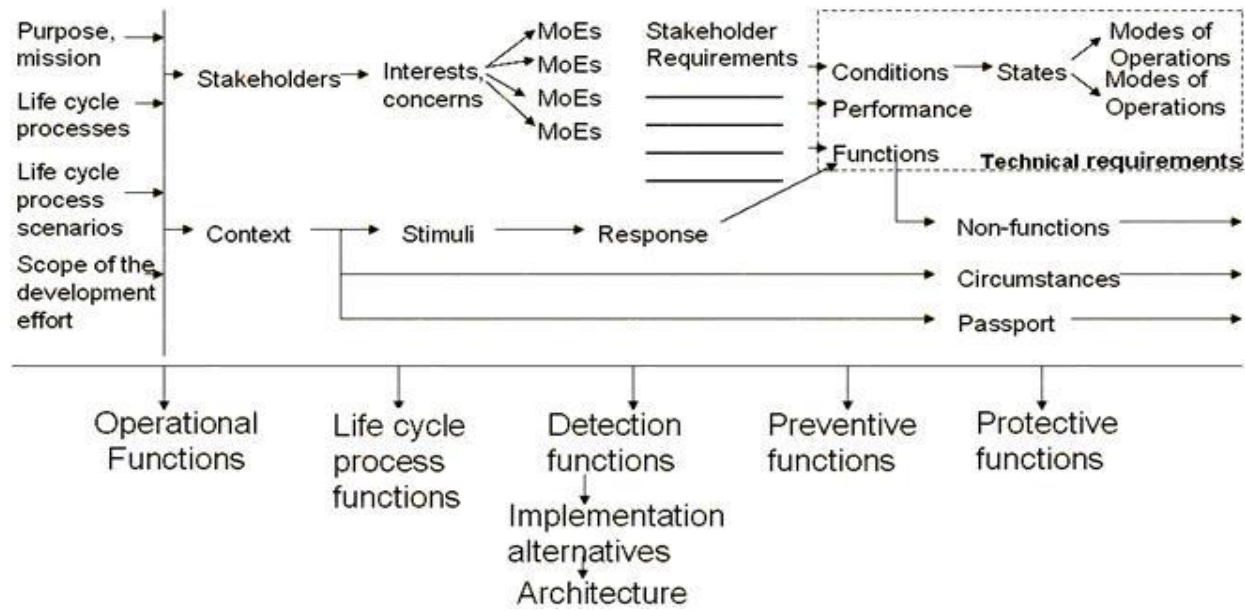

Figure 3. The system concurrent engineering method in detail

\section{The hybrid vehicle system concurrent engineering}

This section illustrates the steps listed in Section 3 highlighting where the proposed approach is different from traditional approaches. The proposed approach is stakeholder driven whereas traditional approaches are customer or user driven. In the various steps listed in Section 3, analysis are performed for each life cycle process scenario, simultaneously, for product and organization. Traditional approaches focus on product operation and development organization. Table 1 presents the life cycle processes and scenarios of a hybrid vehicle. To the processes 'conception', 'system assembly', 'acceleration' and 'maintenance' are considered the cells highlighted in grey as the scope of development effort.

Table 1. Life cycle processes and scenarios

\begin{tabular}{|c|l|l|l|l|}
\hline \multirow{4}{*}{ Processes } & \multicolumn{2}{|c|}{ Organization Processes } & \multicolumn{1}{c|}{ Processes of Product Life Cycle } \\
\cline { 2 - 5 } & Development & $\begin{array}{c}\text { Manufacturing and } \\
\text { Assembly }\end{array}$ & \multicolumn{1}{|c|}{ Operation } & \multicolumn{1}{|c|}{$\begin{array}{c}\text { Support to } \\
\text { Operation }\end{array}$} \\
\hline \multirow{4}{*}{ Scenarios } & Conception & System Assembly & Initialization & Provisioning \\
\cline { 2 - 5 } & Detailed Project & $\begin{array}{l}\text { Manufacturing of } \\
\text { the Structure }\end{array}$ & Neutral & Check-up \\
\cline { 2 - 5 } & $\begin{array}{l}\text { Components } \\
\text { Project }\end{array}$ & $\begin{array}{l}\text { Components } \\
\text { Supplying }\end{array}$ & Acceleration & Maintenance \\
\cline { 2 - 5 } & Prototype & Acceptation Tests & Low Velocity & Towing \\
\cline { 2 - 5 } & Tests & & High Velocity & Repair \\
\hline & Processes of & & Waiting & \\
\hline & Engineering & & & \\
\hline
\end{tabular}

The processes of the life cycle are the ones for which the stakeholder analysis, requirements analysis, functional analysis and implementation architecture analysis 
will be exemplified. In practise steps 1 to 4 in Section 3 must be run for all life cycle process scenarios. Figures 4 to 13 just exemplify the steps for some selected processes.

Figures 4 and 5 exemplify the identification of organization stakeholders for two life cycle processes: 'development' and 'manufacturing and assembly'. The scenarios belongs to the scope of the development effort. This is to show that it is necessary and possible to develop from the outset all processes within the scope of development effort. This innovates the traditional focus on systems engineering the product. This approach recognizes that the system solution is not only made of product elements but also of organization elements. Figures 4 and 5 also captures the stakeholder concerns represented by the connections between the stakeholders and the central bubble, containing the process of the life cicle.

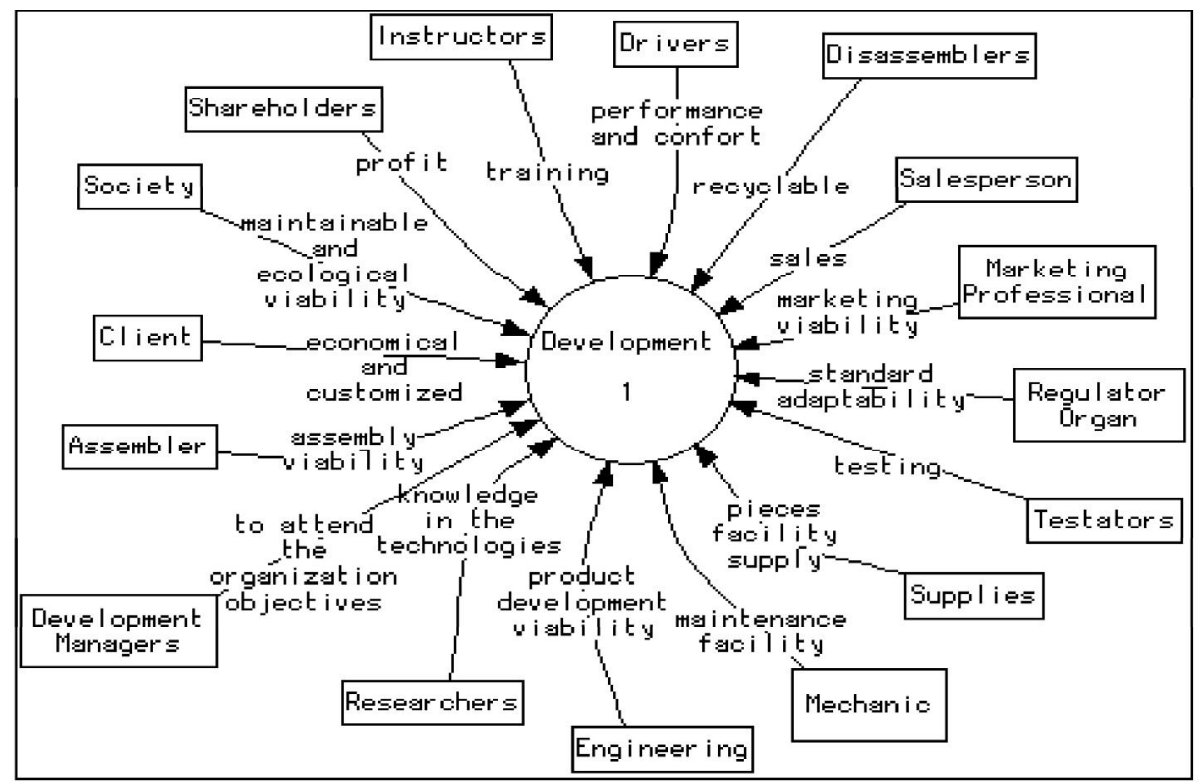

Figure 4. Stakeholders and their concerns for the development analysis

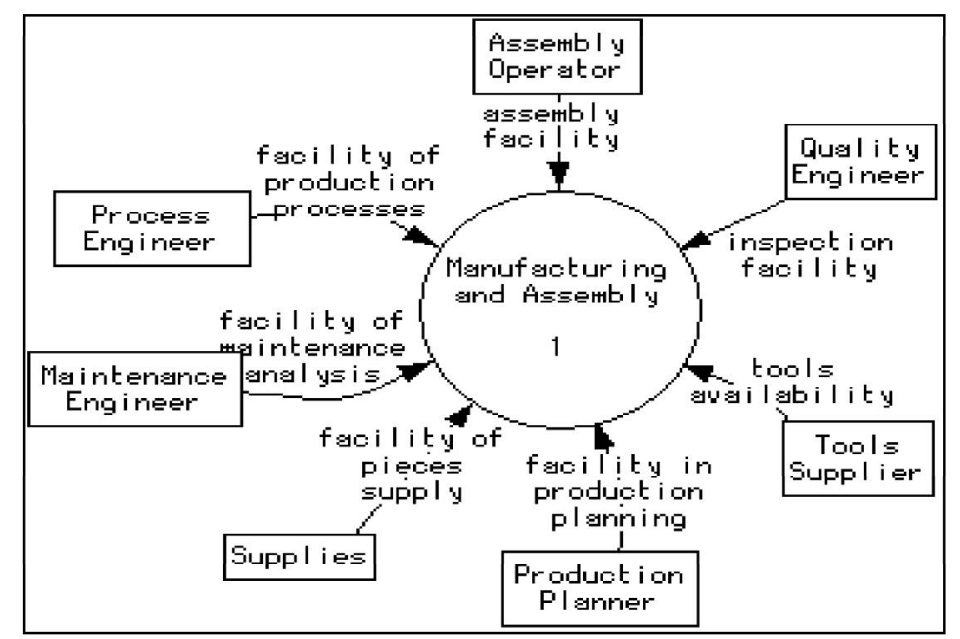

Figure 5. Stakeholders and their concerns for the 'manufacturing and assembly' life cycle process 
Figures 6 and 7 presents the product stakeholders identified and their concerns for two other life cycle processes: 'operation' and 'support to operation'. From stakeholder concerns, stakeholder requirements are identified and measures of effectiveness (MoEs) are derived. From stakeholder requirements, functions, performance and conditions are identified. Requirement analysis transforms stakeholder requirements into system requirements. System requirements will be met not only by product elements but also by organization elements.

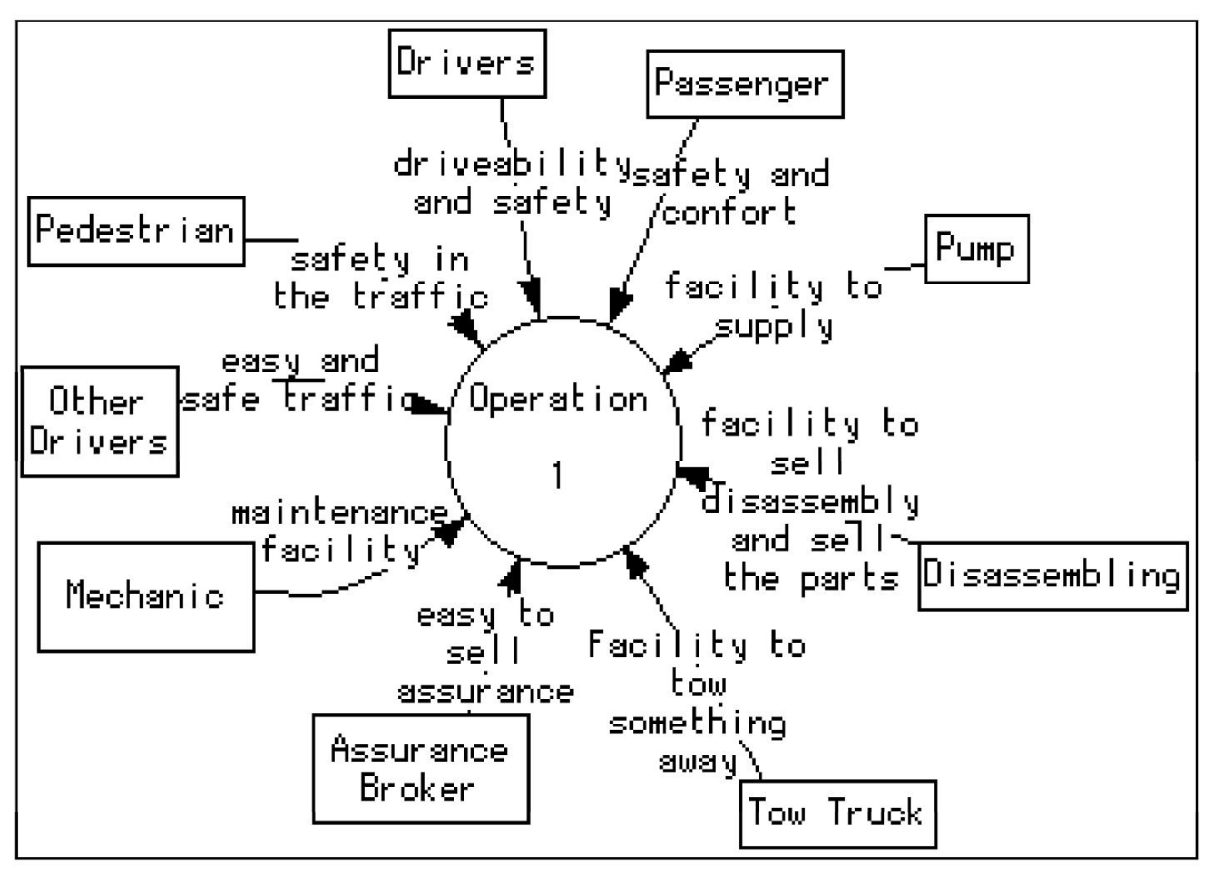

Figure 6. Product stakeholders and their concerns for the 'operation' process

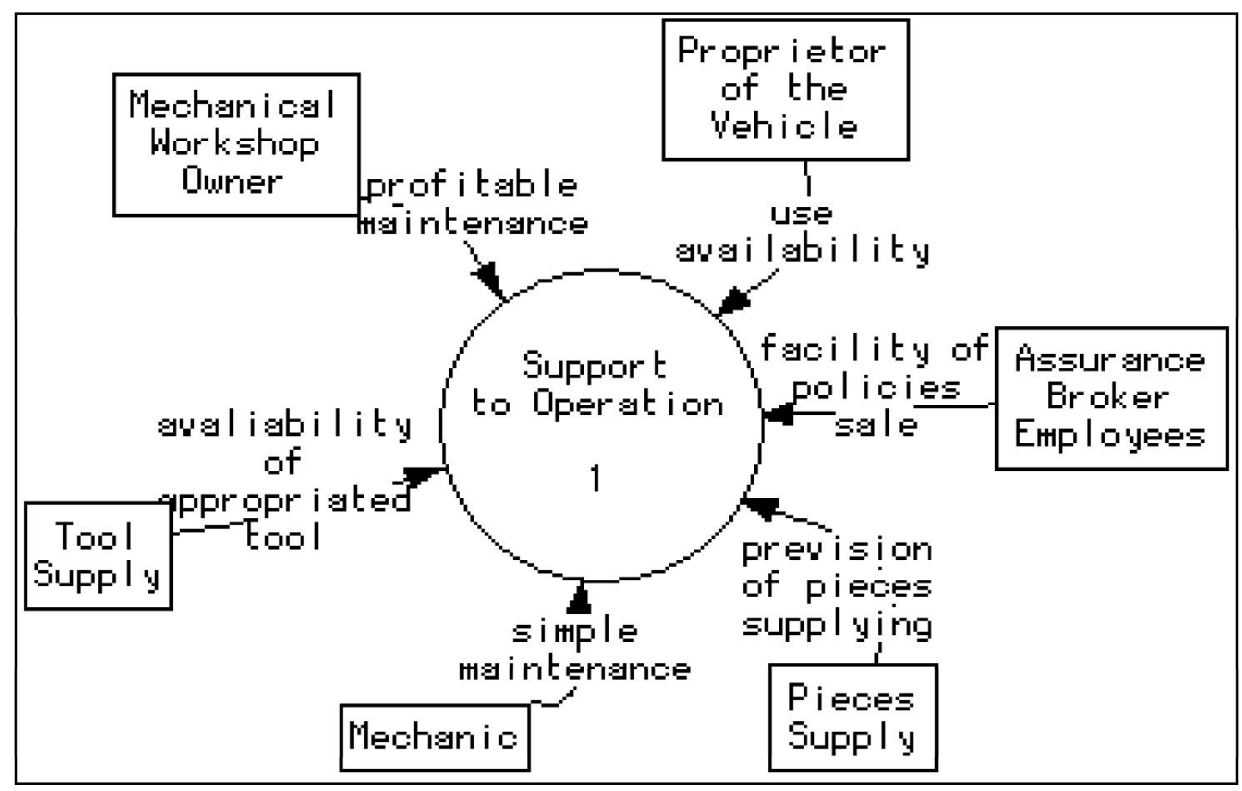

Figure 7. Product stakeholders and their concerns for the 'support to operation' process 
Figures 8 and 9 depict the organization functional context for two life cycle process scenarios: 'conception' and 'assembly'. The links between the central bubble and the elements in the organization environment at that scenario are identified. These links show the flows of information (in this case), material and energy between the environment and the system.

Figures 10 and 11 depict the product during 'providing acceleration' and 'vehicle in maintenance' in the central bubble and the elements in the environment during those processes. Links between product and environment are energy, material and information flows. Besides each element in the environment, some of their potential states is necessary to be identified. In Figure 10 for example, for the 'alimentation system' potential states could be empty. The composition with states of other elements in the environment results in the potential circumstances a system must cope with.

The system must have different modes depending on the circumstances. Behaviour modelling is required to show under which conditions system mode and system state transition occurs. Functions are identified per mode. Functions are identified from outside in by identifying which responses the system is supposed to give to deal with each stimulus provided by the environmental elements. For each function, performance requirements are identified. Circumstances, flows between the system and the environment and function failures are sources of hazards. Risk analysis is performed on each identified potential hazard and exception handling functions are also identified at this stage.

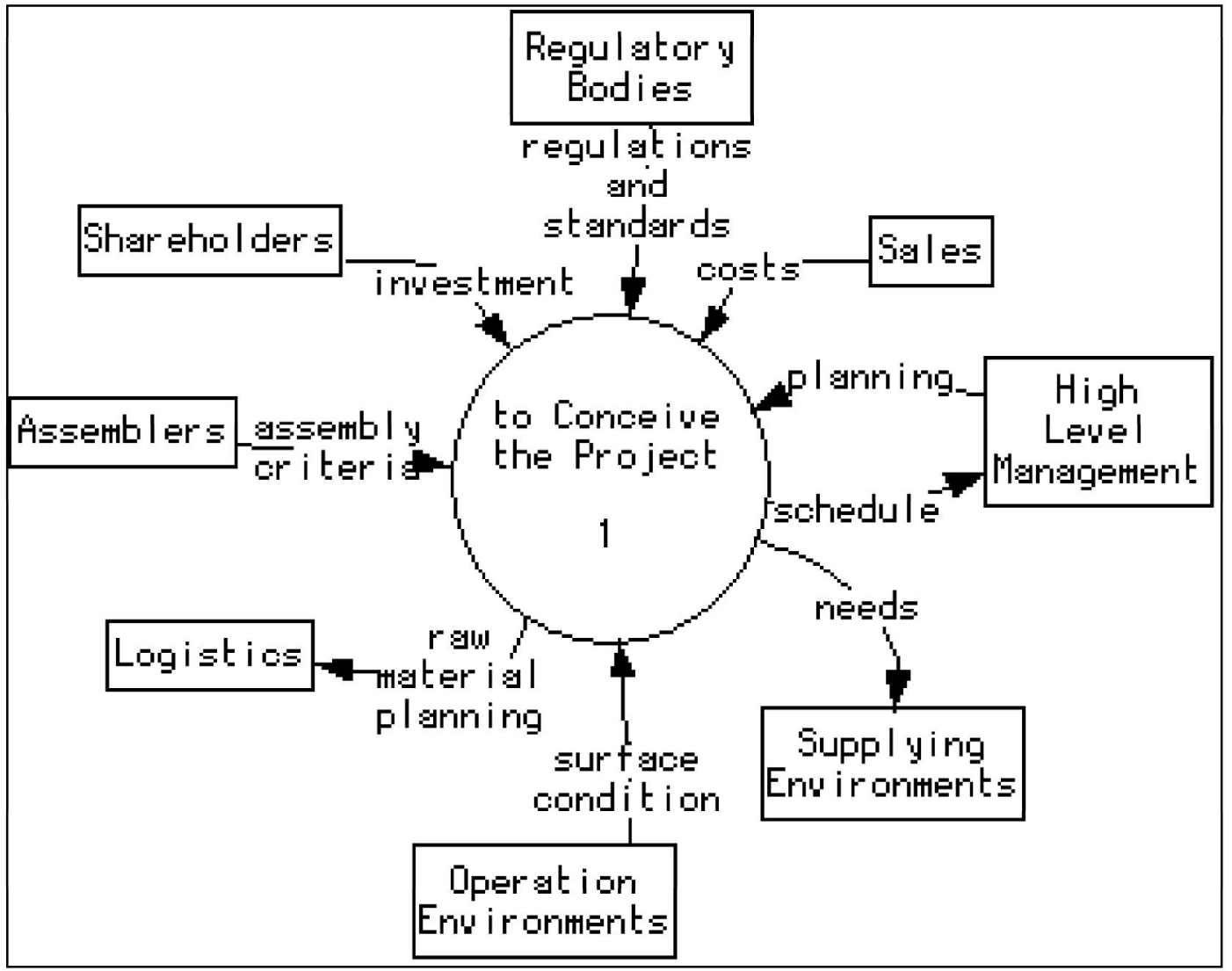

Figure 8. Organization functional interfaces analysis for the 'to conceive the project' scenario 


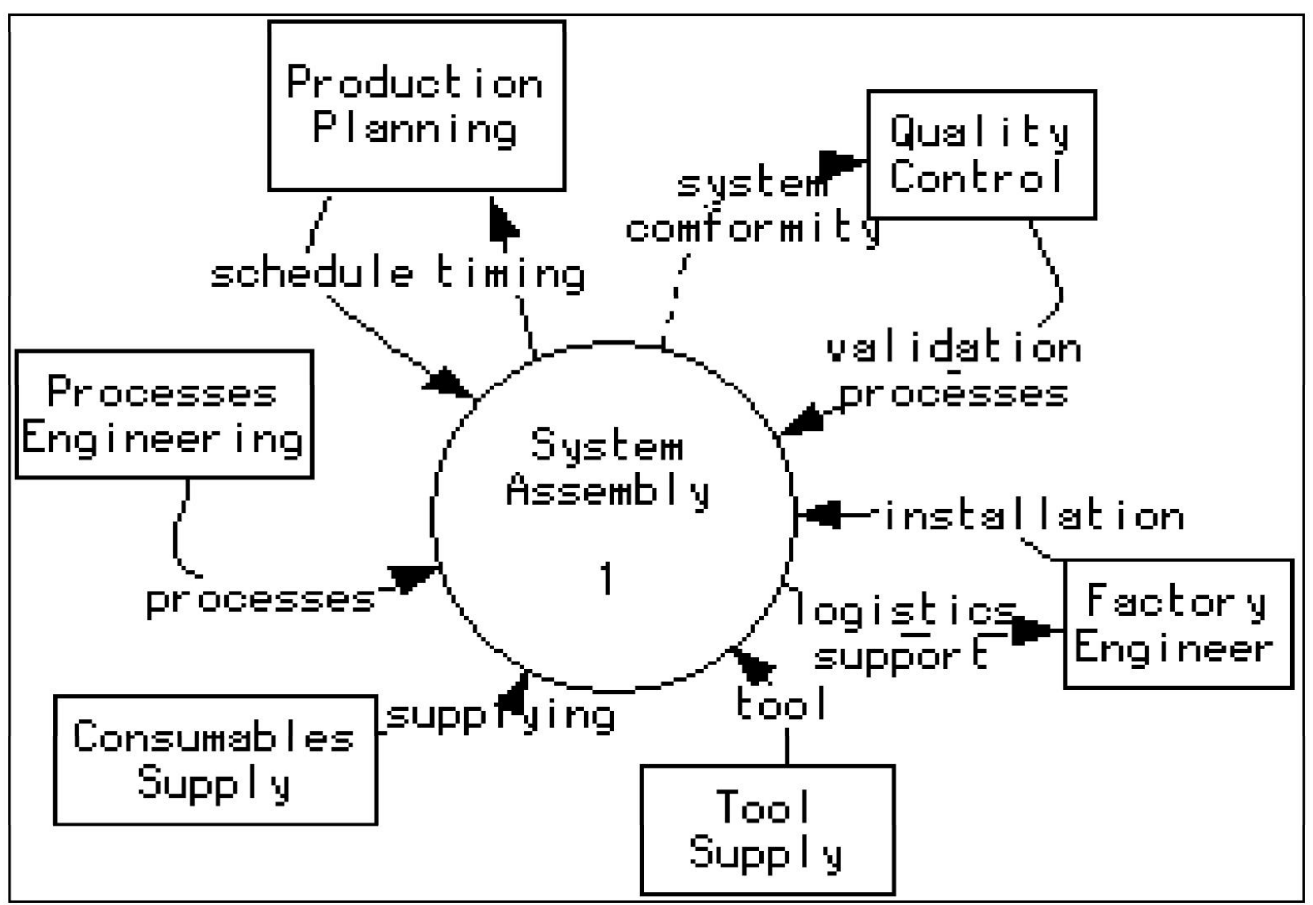

Figure 9. Organization functional interfaces analysis for the 'systems assembly' process scenario

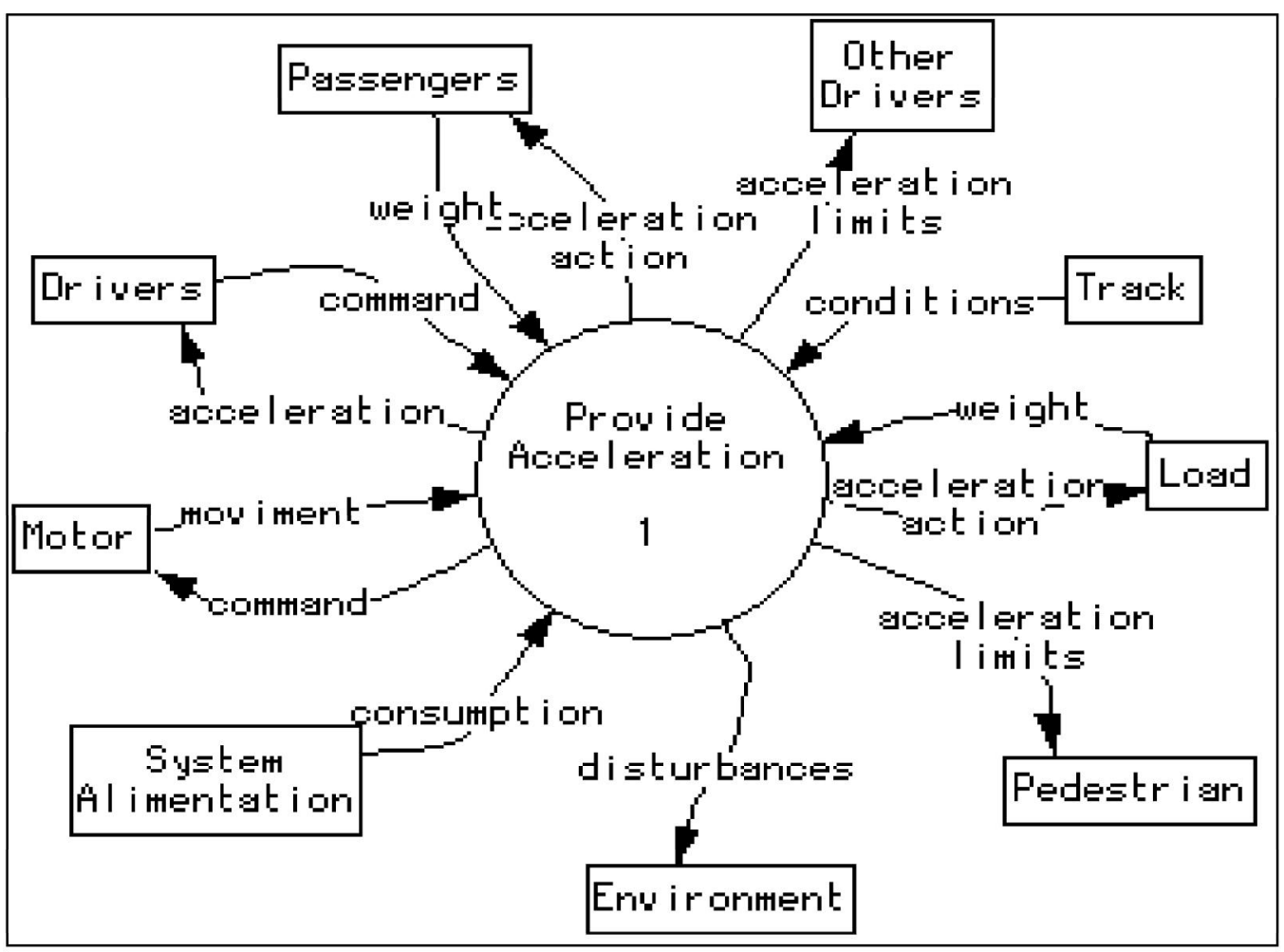

Figure 10. Product functional context for the 'provide acceleration' process scenario 


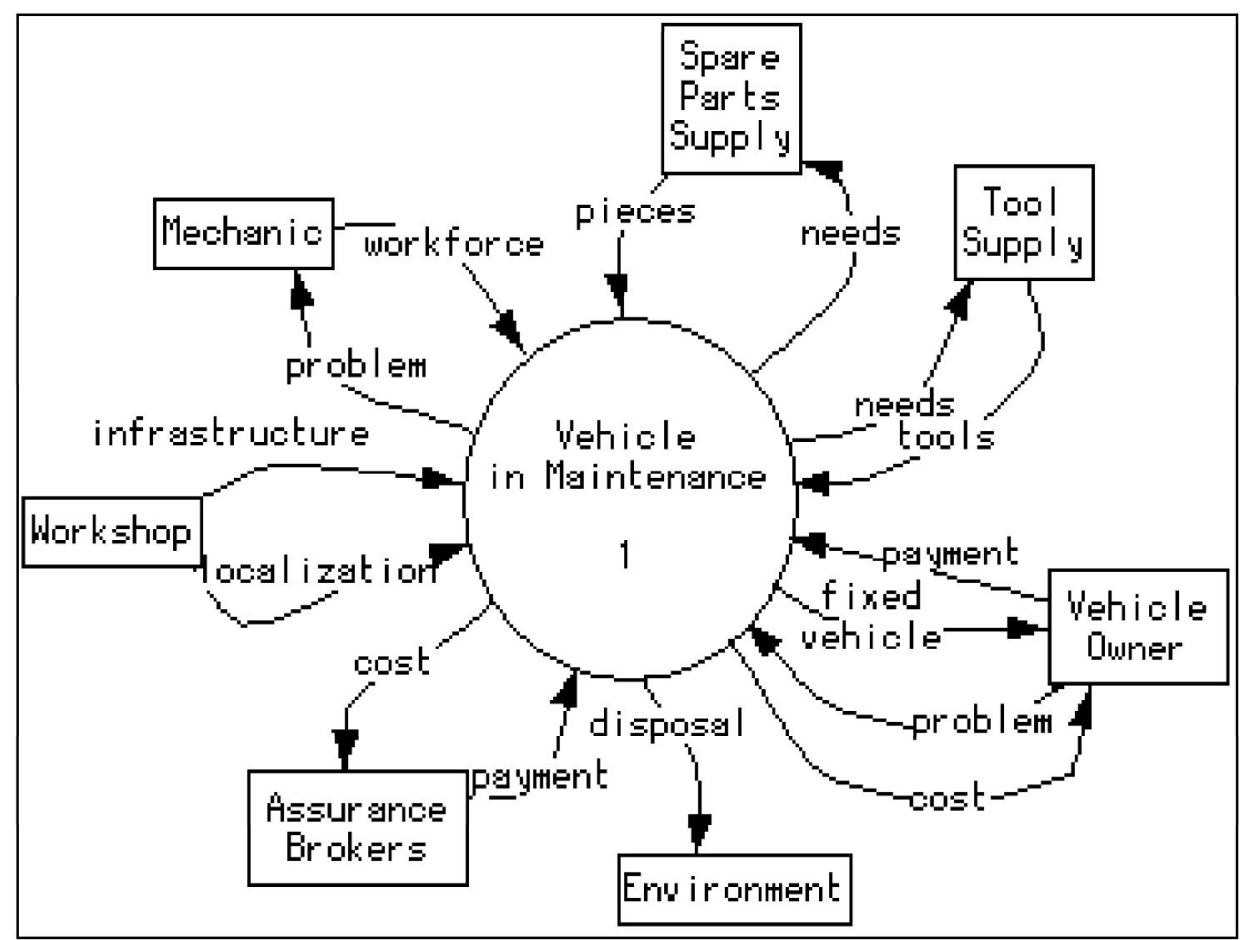

Figure 11. Product functional context for the 'vehicle in maintenance' process

Figure 12 presents the external physical connections and its flows between the elements of the environment and the 'acceleration system'.

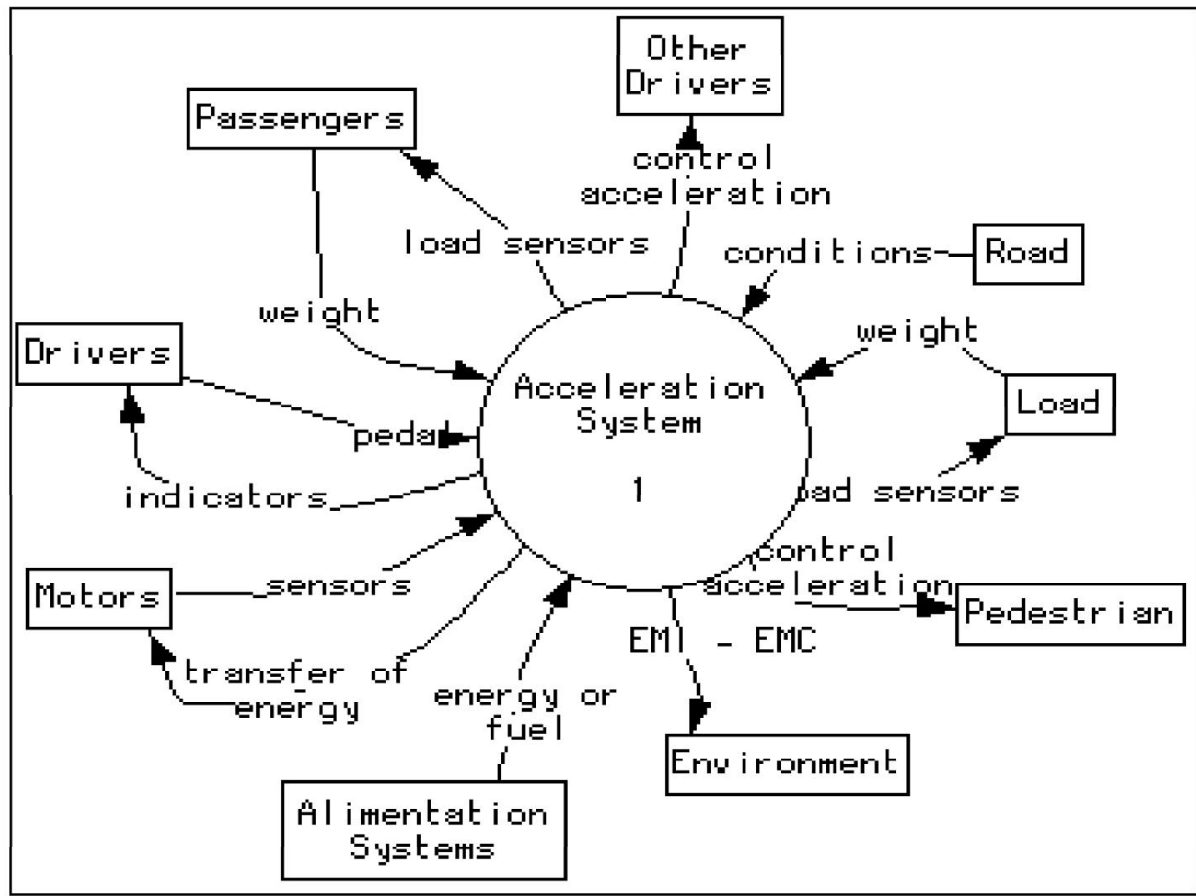

Figure 12. Physical analysis context and external physical interfaces for the 'acceleration system' process 


\section{Discussion}

This sections highlights the differences between traditional and proposed approaches. Complex products such as hybrid vehicle analyzed in this paper have many stakeholders. It is not possible to consider only customer or user as stakeholders of interests, like in the traditional approaches. Stakeholders related to all product life cycle process must be taken into consideration from the outset of the system architecting process. The proposed approach accomplishes it. (see Steps 1 and 2 in Section 3). Traditional systems engineering approaches perform functional context analysis only during product operations (the so called CONOPS or concept of operations) and for product development organization processes. However, a system solution is comprised of product and organization elements and many enabling elements must be also developed for mission success. These elements are only identified if context for each life cycle process scenario is performed. Therefore, the proposed approach covers the overall product life cycle, not only operations and development. (see Step 3 in Section 3). By considering product life cycle processes from the beginning of the system architecting process and from the top level context diagrams to be decomposed in lower level functions and lower level physical architectures, the concurrent engineering concept is implemented within the systems engineering process. This fulfills the framework proposed in Figure 1. The proposed approach allows requirements from the whole product life cycle to be anticipated to the early stages of a system architecting process. Stakeholder requirements are captured for the whole product life cycle process. Functions, performance, conditions, circumstances, modes and exception functions are captured for the whole product life cycle process. External physical and logical interfaces and internal physical and logical interfaces are identified for the whole product life cycle process. The system solution here is composed of product and organization elements. The product interaction with other system elements is identified in the beginning of the system architecting process. This promotes dramatic gains in productivity during product development and during product life cycle. System quality increases. Product changes are avoided. Changes cost and time are eliminated.

\section{Conclusion}

This paper presented a system concurrent engineering approach for the conception of a hybrid vehicle. The proposed approach addressed the deficiencies of traditional methods, such as, product focus, operation and development focus, and part focus. The paper described the approach as a way to perform stakeholder analysis, requirements analysis, functional analysis and implementation architecture, simultaneously, for the product and organization elements of a system at every layer of the system breakdown structure. This is necessary to address all complexity factors that are inherent to complex product development. Conclusions are that impact, traceability and hierarchy links promote the anticipation of life cycle process requirements to the early stages of systems architecting. Late 
changes are avoided, development costs are dramatically reduced while satisfaction of stakeholders over product life cycle is increased.

\section{Acknowledgement}

The authors would like to thank CAPES (Coordination for Supporting and Development Superior Education Personnel, www.capes.br) for Jonas Bianchini Fulindi scholarship.

The authors would like to thank ITA (the Technological Institute of Aeronautics, www.ita.br) for the post graduate course opportunity. The authors would like to thank IAE (Aeronautics and Space Institute, www.iae.cta.br) and 3SL (www.threesl.co.uk) for providing Cradle, the systems engineering environment software used for the development of this work.

\section{References}

[1] Alexander C. Notes on the synthesis of the form. Harvard university press. Cambridge, USA, 1964.

[2] Electronic industries alliance. EIA-632: process for engineering a system. Arligton, 1997.

[3] European cooperation for space standardization. ECSS-E-ST-10C: space engineering: system engineering general requirements. Noordwijk: ESA, 2009.

[4] Hitchins DK. Getting to grips with complexity. In: proceedings of the $2^{\text {nd }}$ annual conference of the incose - UK chapter, 1996.

[5] Huang GQ. Design for x: concurrent engineering imperatives. London: Chapman Hall, 1996.

[6] Institute of electrical and electronics engineers. Systems engineering: application and management of the systems engineering process. New York: IEEE, 2005.

[7] Loureiro G. A systems engineering and concurrent engineering framework for the integrated development of complex products. Ph.D. thesis, Loughborough University, 1999.

[8] National aeronautics and space administration. Nasa systems engineering handbook. Washington, 2007. 